\title{
High Surface Area Ceria Nanoparticles via Hydrothermal Synthesis Experiment Design
}

\author{
Stanislav Kurajica, ${ }^{1}$ Iva Minga, ${ }^{1}$ Martina Guliš, ${ }^{1}$ Vilko Mandić, ${ }^{2}$ and Ivan Simčić ${ }^{3}$ \\ ${ }^{1}$ Faculty of Chemical Engineering and Technology, University of Zagreb, Marulićev Trg 19, 10000 Zagreb, Croatia \\ ${ }^{2}$ Ruđer Bošković Institute, Bijenička Cesta 54, 10000 Zagreb, Croatia \\ ${ }^{3}$ Pliva Croatia d.o.o., Prilaz Baruna Filipovića 25, 10000 Zagreb, Croatia
}

Correspondence should be addressed to Stanislav Kurajica; stankok@fkit.hr

Received 30 March 2016; Revised 21 July 2016; Accepted 21 July 2016

Academic Editor: Ajayan Vinu

Copyright (C) 2016 Stanislav Kurajica et al. This is an open access article distributed under the Creative Commons Attribution License, which permits unrestricted use, distribution, and reproduction in any medium, provided the original work is properly cited.

\begin{abstract}
Hydrothermal synthesis of $\mathrm{CeO}_{2}$ was optimized on two reactant concentrations and synthesis temperature and duration, in order to achieve material having the greatest specific surface area (SSA). Taguchi method of experimental design was employed in evaluation of the relative importance of synthesis parameters. $\mathrm{CeO}_{2}$ nanoparticles were characterized using X-ray diffraction, nitrogen adsorption-desorption isotherms, and scanning electron microscopy. Optimum conditions for obtaining particles with greater SSA were calculated according to Taguchi's model "the-higher-the-better." Synthesis temperature was found to be the only parameter significant for enabling nanoparticles with greater SSA. Mesoporous nanocrystalline ceria with SSA as great as $226 \mathrm{~m}^{2} \mathrm{~g}^{-1}$ was achieved, which is unprecedented for the hydrothermally synthesized ceria. The reason for this achievement was found in temperature dependence of the diffusion coefficient which, when low, favors nucleation yielding with fine particles, while when high it favors crystal growth and formation of one-dimensional structures. The occurrence of 1D-structure in sample exhibiting the smallest SSA was confirmed. Very fine crystallites with crystallite size as low as $5.9 \mathrm{~nm}$ have been obtained being roughly inverse proportional to SSA. Selected samples were tested as catalyst for soot oxidation. Catalyst morphology turned out to be decisive factor for catalytic activity.
\end{abstract}

\section{Introduction}

Ceria, $\mathrm{CeO}_{2}$, has been investigated as catalyst for various industrial and environmental applications [1]. Recently, the investigations have focused on nanostructured ceria and its properties and synthesis methods [2] since it has been shown that nanostructured ceria is superior to bulk ceria in terms of both catalysis and support [3]. Different synthesis approaches have been used for synthesis of ceria nanoparticles $[2,4$, 5] among which hydrothermal synthesis draws significant interest [2, 6-21].

Hydrothermal synthesis possesses numerous advantages of one-pot, one-step, low-temperature, low-cost, and environmentally benign process enabling preparation of high purity nanoparticles of desired size and morphology [1113]. Early investigations on hydrothermal synthesis for the preparation of $\mathrm{CeO}_{2}$ powders, such as those conducted by Hirano and Kato [14, 15], have been concentrated on various process precursors. Shortly afterwards, experimental conditions, specifically precursor concentrations and reaction time and temperature, were studied $[16,17]$. Later on, focus in hydrothermal synthesis studies shifted from particle size to morphology, and ceria with various shapes such as nanorods, nanotubes, nanoplates, nanocubes, and hollow nanospheres has been prepared using hydrothermal method [11, 18, 19]. Finally, $\mathrm{CeO}_{2}$ nanostructured materials doped with various elements have been prepared [20,21].

One of the most beneficial properties for catalytic application is the large surface area. Commercial ceria specific surface area ranges below $10 \mathrm{~m}^{2} \mathrm{~g}^{-1}[7,22]$, while reports on SSA as high as $277 \mathrm{~m}^{2} \mathrm{~g}^{-1}$ could be found in literature for ceria prepared using combined sol-gel and solvothermal process [22], 
TABLE 1: Process variables (factors) used.

\begin{tabular}{lcccc}
\hline Assignation & Variable & Unit & $\begin{array}{c}\text { Lower } \\
\text { value, level 1 }\end{array}$ & $\begin{array}{c}\text { Higher } \\
\text { value, level 2 }\end{array}$ \\
\hline$A$ & $c(\mathrm{NaOH})$ & $\mathrm{mol} / \mathrm{L}$ & 8 & 12 \\
$B$ & $n\left(\mathrm{Ce}\left(\mathrm{SO}_{4}\right)_{2} \times 3 \mathrm{H}_{2} \mathrm{O}\right)$ & $\mathrm{mmol}$ & 0.8 & 1.2 \\
$C$ & Temperature & ${ }^{\circ} \mathrm{C}$ & 120 & 180 \\
$D$ & Duration & $\mathrm{h}$ & 16 & 24 \\
\hline
\end{tabular}

TABLE 2: Experimental layout defined based on Taguchi L8 orthogonal array.

\begin{tabular}{lllll}
\hline Experiment number & $A$ & $B$ & $C$ & $D$ \\
\hline 1 & 1 & 2 & 1 & 1 \\
2 & 2 & 1 & 2 & 1 \\
3 & 2 & 2 & 1 & 2 \\
4 & 2 & 1 & 1 & 1 \\
5 & 2 & 2 & 2 & 2 \\
6 & 1 & 1 & 1 & 2 \\
7 & 1 & 1 & 2 & 2 \\
8 & 1 & 2 & 2 & 1 \\
\hline
\end{tabular}

$200 \mathrm{~m}^{2} \mathrm{~g}^{-1}$ for precipitation process [23], and $139 \mathrm{~m}^{2} \mathrm{~g}^{-1}$ for hydrothermally synthesized ceria [10].

The Taguchi method of experimental design is a method for testing the relative importance of parameters in the experiment outcome. This approach is considered as very efficient since a lot of information is obtained from a reduced set of experiments [24].

This study employs the experiment design in order to yield nanostructured $\mathrm{CeO}_{2}$ having favorable properties using hydrothermal method of synthesis. Thereby, the relative effect of reactants concentrations, synthesis temperature, and duration on the specific surface area, as well as on other properties of ceria, was examined.

\section{Materials and Methods}

In the course of the hydrothermal synthesis of ceria nanoparticles, four process parameters were detected as potentially significant: concentration of two precursors, $\mathrm{NaOH}$ and $\mathrm{Ce}\left(\mathrm{SO}_{4}\right)_{2} \times 3 \mathrm{H}_{2} \mathrm{O}$, and temperature and duration of synthesis. In order to examine their effect on the properties of ceria nanoparticles a Taguchi experimental design has been employed. Four factors (process variables) at two levels for each factor (Table 1) were selected. A common experimental design including combinations of all input factors (fullfactorial design) would require $2^{4}=16$ experiments. On the other hand, Taguchi orthogonal design requires only 8 experiments, half of full-factorial design. L8 orthogonal array (Table 2) is generated using JMP computer program for statistical analysis (JMP ${ }^{\circledR}$, Version 11, SAS Institute Inc., Cary, NC, 1989-2013).

The typical synthesis procedure was as follows: appropriate amount of $\mathrm{Ce}\left(\mathrm{SO}_{4}\right)_{2} \times 3 \mathrm{H}_{2} \mathrm{O}$ (Merck, Germany) has been dissolved in $56 \mathrm{~cm}^{3}$ of $\mathrm{NaOH}$ (Kemika, Croatia) solution and put in a $70 \mathrm{~mL}$ capacity Teflon-lined stainless-steel autoclave, sealed tightly, and thermally treated in temperaturecontrolled oven. Eight samples were prepared; the synthesis conditions used are shown in Tables 1 and 2. After cooling of the autoclave, the product was centrifuged and obtained pale yellow precipitate was washed with demineralized water with the aid of sonication. Centrifugation and washing sequences have been repeated three times. Finally, yielded materials were dried at $60^{\circ} \mathrm{C}$ for $24 \mathrm{~h}$ in static air.

The crystal phase in samples was identified by powder Xray diffraction (XRD) analysis using Shimadzu XRD 6000 diffractometer with $\mathrm{CuK} \alpha$ radiation operated at $40 \mathrm{kV}$ and $30 \mathrm{~mA}$. Data were collected between 5 and $70^{\circ} 2 \theta$ in a step scan mode with steps of $0.02^{\circ}$ and counting time of $2 \mathrm{~s}$. The crystallite size was estimated from XRD peak broadening using Scherrer's formula:

$$
D=\frac{k \lambda}{\beta \cos \theta},
$$

where $D$ is crystallite size, $k$ is shape factor, $\lambda$ is $\mathrm{CuK} \alpha$ radiation wavelength, $\beta$ is peak full width at half maximum corrected for instrumental broadening, and $\theta$ is Bragg angle.

Surface properties of samples were determined by a Brunauer-Emmet-Teller (BET) $\mathrm{N}_{2}$ gas adsorption-desorption isotherms obtained on Micromeritics ASAP-2000 at $77 \mathrm{~K}$. Samples were previously degassed at $100^{\circ} \mathrm{C}$ under a dynamic vacuum of $13 \mathrm{mPa}$ to remove any surface adsorbed residues. Surface area was calculated by utilizing the desorption data. Pore size and pore volume were calculated by BJH method applied to the desorption branch of the isotherms.

Morphology of the samples was investigated using field emission gun scanning electron microscopy (FESEM) device JEOL model 7000F. The samples were sputtered with gold by Quorum SC 7620 sputter coater.

The catalytic efficiency of the samples for soot oxidation was examined according to the method of Sudarsanam et al. [25] where $10 \pm 1 \mathrm{mg}$ of catalyst was mixed with $10 \pm 1 \mathrm{mg}$ of Norit DLC Super 30 carbon black (Cabot Norit Nederland $\mathrm{BV}$ ) in $\alpha$-alumina crucible with spatula for $2 \mathrm{~min}$ and placed in a thermogravimetric analyzer (TGA) Netzsch STA409. Oxidation experiment consisted of heating the catalyst-soot mixture at a rate of $10 \mathrm{~K} \mathrm{~min}^{-1}$ from ambient temperature to $1300^{\circ} \mathrm{C}$ under a $100 \mathrm{~cm}^{3} \mathrm{~min}^{-1}$ synthetic air purge gas flow. Each test was repeated three times to ensure the reproducibility of the obtained results.

\section{Results and Discussion}

The powder XRD patterns (Figure 1) of the prepared samples are indexed to ceria, $\mathrm{CeO}_{2}$ (ICDD PDF number 34-394). Ceria adopts the fluorite crystal structure with space group Fm-3m. In FCC ceria unit cell $\mathrm{Ce}^{4+}$ ions are close packed while $\mathrm{O}^{2-}$ ions occupy tetrahedral sites. Beside peak angles, peak intensities also obey the profile of ICDD PDF number 34-394. Broad peaks point out to formation of nanocrystalline particles. The differences among patterns presented in Figure 1 indicate that different synthesis conditions affect the crystallite size of $\mathrm{CeO}_{2}$. However, no significant difference 
TABLE 3: Average crystallite size calculated from broadening of (220) XRD reflection using Scherrer's formula and specific surface area calculated from desorption isotherms by the $\mathrm{BJH}$ model.

\begin{tabular}{lccc}
\hline Sample & $\begin{array}{c}\text { Crystallite } \\
\text { size }(\mathrm{nm})\end{array}$ & $\begin{array}{c}\text { Specific surface } \\
\text { area }\left(\mathrm{m}^{2} \mathrm{~g}^{-1}\right)\end{array}$ & $\begin{array}{c}\text { Dominant pore } \\
\text { radius }(\mathrm{nm})\end{array}$ \\
\hline CNR1 & $5.9 \pm 0.3$ & $181.7 \pm 0.5$ & 3.3 \\
CNR2 & $14.3 \pm 1.0$ & $108.6 \pm 1.0$ & 6.2 \\
CNR3 & $7.5 \pm 0.5$ & $175.0 \pm 1.9$ & $3.2,6.3$ \\
CNR4 & $6.5 \pm 0.4$ & $178.9 \pm 1.5$ & 3.2 \\
CNR5 & $21.4 \pm 1.5$ & $65.1 \pm 0.8$ & 6.3 \\
CNR6 & $6.2 \pm 0.4$ & $226.7 \pm 1.1$ & 3.3 \\
CNR7 & $12.3 \pm 0.9$ & $114.6 \pm 1.5$ & 3.7 \\
CNR8 & $12.5 \pm 0.8$ & $106.7 \pm 1.1$ & 3.6 \\
\hline
\end{tabular}

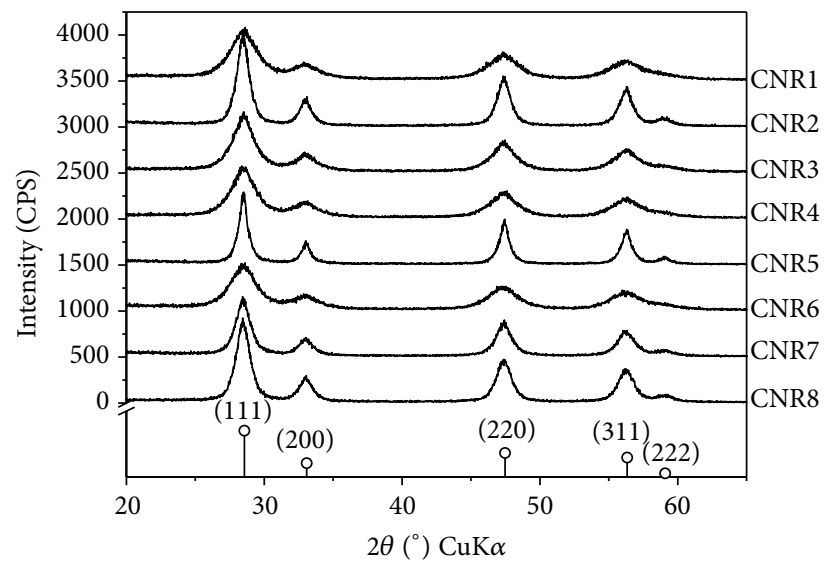

FIGURE 1: Powder XRD patterns of the prepared samples.

in background noise has been noted. Excessive background noise would be a presence indication of great amount of amorphous phase, that is, poor crystallinity.

The only peak that is not partially overlapped, reflection (220), was the basis for the average crystallite size calculation. The crystallite sizes calculated using Scherrer's formula are given in Table 3. As can be seen, sample 5 presents the greatest, while sample 6 have the smallest crystallite size.

The nitrogen adsorption-desorption isotherms of the prepared samples are illustrated in Figure 2(a) (samples CNR1, CNR3, CNR4, and CNR6) and Figure 2(b) (samples CNR2, CNR5, CNR7, and CNR8). According to the IUPAC classification isotherms presented in Figure 2(b) could be without doubt classified as type IV [26]. Shape of isotherms presented in Figure 2(a) does not allow such straightforward classification. A typical feature of type IV isotherms is final saturation plateau of variable length, despite being sometimes reduced to inflection point [26]. As can be seen, no plateau could be observed in isotherms depicted in Figure 2(a). It could be speculated that isotherms presented in Figure 2(a) resemble to type II isotherms. However, the occurrence of hysteresis loop is not consistent with this type of isotherms. Hysteresis is a consequence of capillary condensation [26] (i.e., the initial monolayer-multilayer adsorption on the walls of mesopores is followed by pore condensation), while type
II isotherms are associated with nonporous adsorbents [26]. On the other hand, completely reversible isotherms, having no hysteresis at all, are rarely observable. After careful consideration of presented arguments, despite not being typical, isotherms in Figure 2(a) are classified as type IV. Zagaynov and Kutsev [27] in their study on ceria nanopowders also classified isotherms similar to the ones depicted in Figure 2(a) as type IV.

The hysteresis loop shapes are generally related to pores structure. Narrow hysteresis loops of isotherms presented in Figure 2(a) (samples CNR1, CNR3, CNR4, and CNR6) could be classified as $\mathrm{H} 3$ type while broader loops presented in Figure 2(b) (samples CNR2, CNR5, CNR7, and CNR8) are of H2 type [26]. H3 loops are often associated with nonrigid aggregates of plate-like particles or pore network that consisted of macropores [26]. H2 loops are given by complex pore structures, very often in solids made by aggregates or agglomerates of spheroidal particles of nonuniform size and shape [5].

The BET specific surface areas of the prepared samples are given within Table 3. As can be seen, the specific surface areas vary significantly with the preparation conditions. Among the prepared samples, sample 5 provided the lowest and sample 6 provided the highest specific surface area. Obtained specific surface areas corroborate well with the previously determined average crystallite sizes being inversely proportional. To the best of our knowledge, for the ceria prepared using hydrothermal synthesis, SSA as great as $226 \mathrm{~m}^{2} \mathrm{~g}^{-1}$ was not reported before. It is important to stress that such favorable outcome has been achieved without any additive, using just plane hydrothermal process while adjusting only the reaction parameters. In such manner no organic contaminants, which can reduce catalytic activity of ceria, were introduced.

The pore size distributions curves were calculated from desorption branch of the isotherms by the $\mathrm{BJH}$ model. Dominant pore radiuses are presented within Table 3. All samples but sample 3 have monomodal pore size distribution while sample 3 displays bimodal pore size distribution. All pore diameters are within the range of mesopores which is consistent with the existence of nanoparticles aggregates.

Specific surface area was taken as the output of the experiment. In order to determine the effect of each variable on the output, the signal-to-noise (SN) ratio of the Taguchi approach has to be calculated for each experiment. There are three categories of performance characteristics, that is, the-lower-thebetter, the-higher-the-better, and the-nominal-the-better. In this particular case maximizing the performance characteristic, that is, the-higher-the-better, is required and therefore specific surface area values were transformed into the signalto-noise ratio using the following equation:

$$
\mathrm{SN}=-10 \log \left[\frac{1}{m} \sum_{i=1}^{m} \frac{1}{y_{i}^{2}}\right],
$$

where $m$ is the number of trials for each experiment and $y_{i}$ is the mean value of the observed performance characteristic for a given experiment. The SN ratio values for all samples calculated on the basis of (2) are given in Table 4 .

Using Taguchi experimental design approach, it is possible to separate out the effect of each factor at each level [28]. 


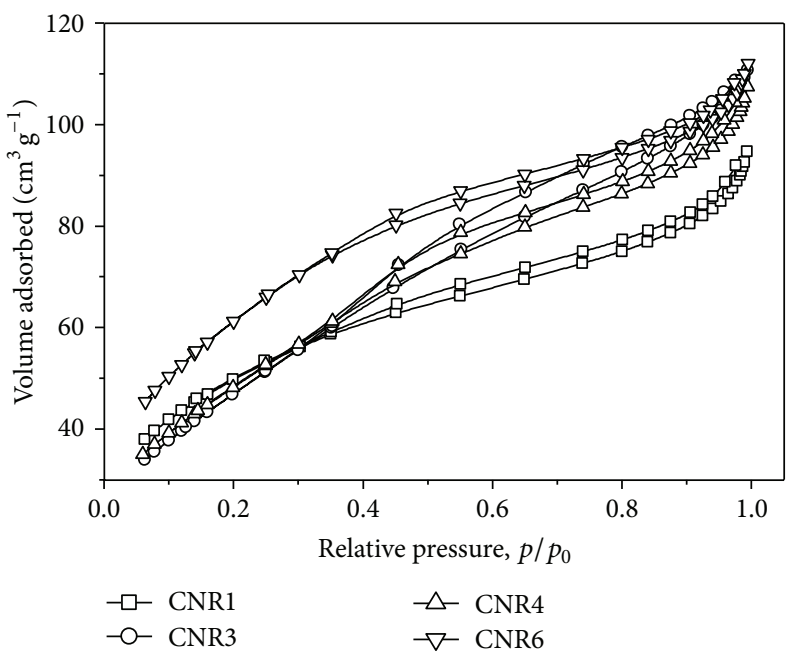

(a)

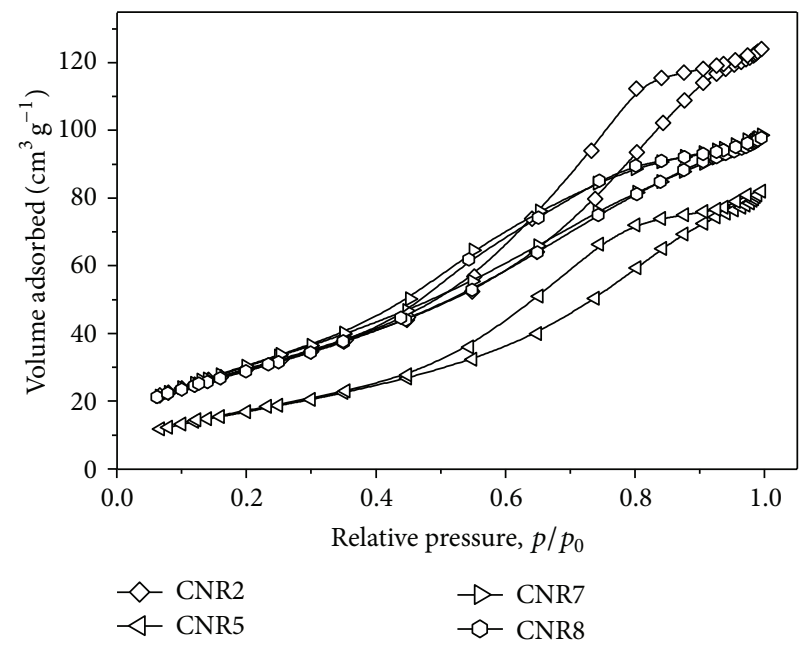

(b)

FIGURE 2: Nitrogen adsorption-desorption isotherms of the prepared samples.

TABLE 4: Signal-to-noise ratio according to (2) for each experiment.

\begin{tabular}{lcccccccc}
\hline Experiment & 1 & 2 & 3 & 4 & 5 & 6 & 7 & 8 \\
\hline SN ratio & 45.19 & 40.72 & 44.86 & 45.05 & 36.27 & 47.11 & 41.18 & 40.56 \\
\hline
\end{tabular}

TABLE 5: SN ratio for each factor and level as well as influence of each factor to specific surface area.

\begin{tabular}{lccc}
\hline Factor & $\mathrm{SN}_{1}$ & $\mathrm{SN}_{2}$ & $R$ \\
\hline$A$ & 43.51 & 41.73 & 1.78 \\
$B$ & 43.52 & 41.72 & 1.80 \\
$C$ & 45.56 & 39.68 & 5.88 \\
$D$ & 42.88 & 42.36 & 0.52 \\
\hline
\end{tabular}

First, the average $\mathrm{SN}$ ratio value is calculated for each factor and level. For example, the average $\mathrm{SN}$ ratio values for factor $A$ (concentration of $\mathrm{NaOH}$ ) at level $1\left(8 \mathrm{~mol} \mathrm{~L}^{-1}\right)$ can be calculated by averaging $\mathrm{SN}$ ratios from experiments 1, 6, 7, and 8:

$$
\mathrm{SN}_{A 1}=\frac{\mathrm{SN}_{1}+\mathrm{SN}_{6}+\mathrm{SN}_{7}+\mathrm{SN}_{8}}{4} .
$$

The influence of factor is defined as the absolute difference between the average $\mathrm{SN}$ ratios of the two levels. For example, the influence of factor $A$ is the absolute difference between the effect of factor $A$ at level $1, \mathrm{SN}_{A 1}$, and the effect of factor $A$ at level $2, \mathrm{SN}_{A 2}$ :

$$
R_{A}=\left|\mathrm{SN}_{A 1}-\mathrm{SN}_{A 2}\right| .
$$

The larger the influence of factor, $R$, the larger the effect that the variable has on the observed performance [28]. SN ratio values for each factor and level, as well as influence of each factor, are tabulated in Table 5.

Factor effect plots were used to visualize performance changes as each individual factor level is changed (Figure 3). The slope of the line indicates the impact magnitude of particular factor on specific surface area. Based on Figure 4, it could be estimated that the most influential factor was the temperature of hydrothermal synthesis.

In order to determine if the influence of any factor on specific surface area is statistically significant, the analysis of variance (ANOVA) has been performed. Generally, ANOVA is statistical technique for estimating the degree of difference or similarity between two or more groups of data. Here, oneway ANOVA (JMP, Version 11, SAS Institute Inc., Cary, NC, 1989-2013) providing calculation of the significance level of each process parameter on observed performance was used.

From the $\mathrm{SN}$ ratios, the overall $\mathrm{SN}$ ratio is expressed as

$$
\overline{\mathrm{SN}}=\frac{1}{n} \sum_{1}^{n} \mathrm{SN}_{n}
$$

where $n$ is the number of experiments according to orthogonal array, $\overline{\mathrm{SN}}$ is the overall mean of $\mathrm{SN}$ ratio, and $\mathrm{SN}_{n}$ is the $\mathrm{SN}$ ratio for $n$th experiment. In that manner, the overall mean of $\mathrm{SN}$ ratio was calculated to be 42.62 .

The total sum of squares of signal-to-noise ratio due to variation about overall mean is

$$
\mathrm{SS}_{t}=\sum_{n=1}^{n}\left(\mathrm{SN}_{n}-\overline{\mathrm{SN}}\right)^{2}
$$

The value of $\mathrm{SS}_{t}$ is given in Table 6.

The sum of squares for each factor due to variation about overall mean is

$$
\mathrm{SS}_{i}=\frac{n}{i} \sum_{j=1}^{j}\left(\mathrm{SN}_{i j}-\overline{\mathrm{SN}}\right)^{2}
$$

where $i$ denotes factor and $j$ denotes level so $\mathrm{SN}_{i j}$ is the average $\mathrm{SN}$ ratio of $i$ th parameter of $j$ th level. The values of $\mathrm{SS}_{i}$ have been given in Table 6. 

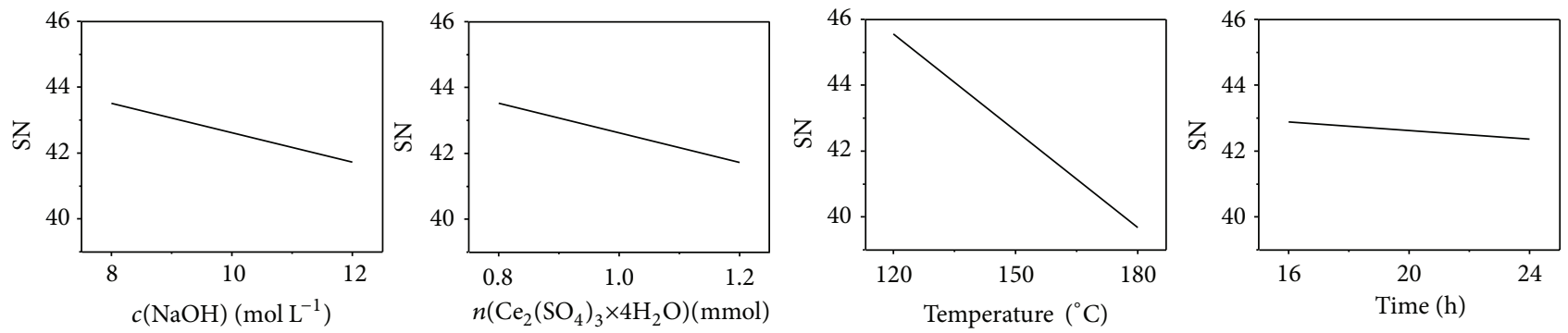

FIgURE 3: Factor effects plots for each individual factor level.

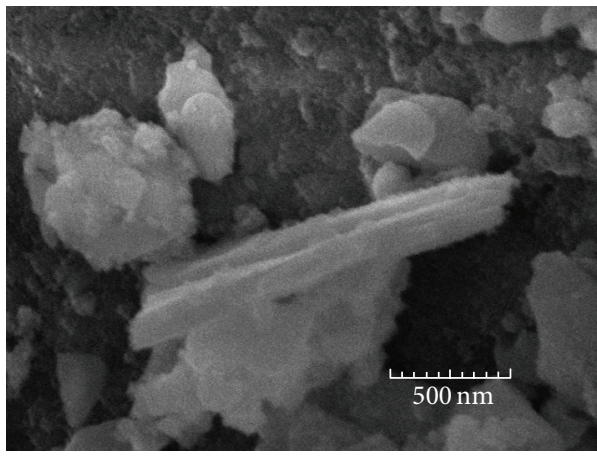

(a)

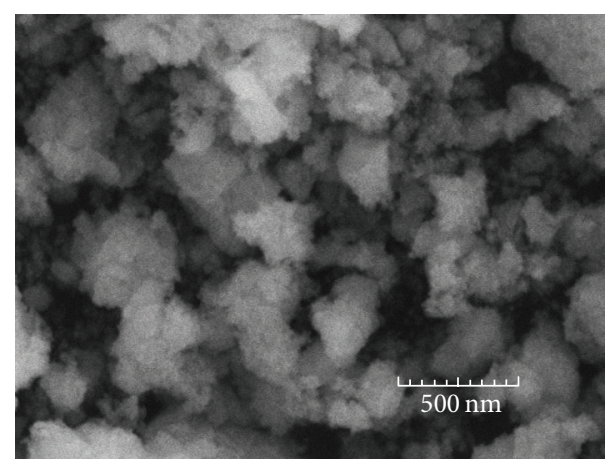

(b)

Figure 4: FESEM micrographs of (a) sample CNR5 and (b) sample CNR6.

TABLE 6: Summary of ANOVA for SN ratio.

\begin{tabular}{lccccc}
\hline Factor & DoF & $\mathrm{SS}_{i}$ & Mean SS $_{i}$ & $F$-ratio & $p$ value \\
\hline$A, c(\mathrm{NaOH})$ & 1 & 6.38 & 6.38 & 3.38 & 0.16 \\
$B, c\left(\mathrm{Ce}\left(\mathrm{SO}_{4}\right)_{2} \times 3 \mathrm{H}_{2} \mathrm{O}\right)$ & 1 & 6.44 & 6.44 & 3.42 & 0.16 \\
$C$, temperature & 1 & 68.89 & 68.89 & 36.54 & 0.01 \\
$D$, duration & 1 & 0.55 & 0.55 & 0.29 & 0.63 \\
$\mathrm{SS}_{e}$ & 3 & 5.66 & 1.89 & & \\
$\mathrm{SS}_{t}$ & 7 & 87.92 & & & \\
\hline
\end{tabular}

The sum of squares of the errors correlated to all factors is

$$
\mathrm{SS}_{e}=\mathrm{SS}_{t}-\sum_{i=1}^{n} \mathrm{SS}_{i} .
$$

The sum of squares of the errors is given in Table 6 .

The mean square for each factor is obtained by dividing the sum of squares by the degrees of freedom (Table 6). In similar manner, the mean square of the error is obtained by dividing the sum of squares of the residual error by the degrees of freedom (Table 6).

Dividing the mean square for each factor by the mean square error gives $F$-ratio:

$$
F=\frac{{\text { Mean } S_{i}}_{\text {Mean } S_{e}}}{\text { Me }}
$$

A very large $F$-ratio means that the effect variance exceeds the error variance by a substantial amount.
In order to determine whether any of the differences between the means are statistically significant, $p$ values for each parameter have to be calculated and compared to significance level. Large $p$ value suggests that data do not significantly differ, but if $p$ value is small, it is likely that the observed difference is practically significant. Usually, a significance level of $\alpha=0.05$ is used as a boundary value indicating a $5 \%$ risk of identifying a difference when actually none exists.

The ANOVA results for $\mathrm{SN}$ ratio of achieving the greatest specific surface area are given in Table 6. The analysis of the results showed that for achieving great SSA the only significant parameter is the temperature of the thermal treatment while the influences of the other three parameters are statistically irrelevant. In order to achieve great SSA, the temperature of thermal treatment should be held at $120^{\circ} \mathrm{C}$. It has to be stressed that these findings are based solely on the factor levels considered in this study and may vary if different factor levels are used.

The reasons why samples prepared at lower temperatures have greater specific surface areas and smaller crystallite sizes are worth discussion. Hydrothermal synthesis of ceria quite commonly yields one-dimensional structures $[2,7,9]$. It is believed that in the course of hydrothermal and solvothermal synthesis diffusion coefficient is the key factor for the formation of 1D-nanostructured materials [29]. Any crystallization from solution involves two steps: nucleation and growth. Low diffusion coefficient benefits nucleation while high diffusion coefficient is beneficial for crystal growth and may influence the preferred growth along certain direction [29]. The intense nucleation further yields $0 D$ nanoparticles, while growth, due 
to enhanced diffusion coefficient, produces $1 \mathrm{D}$ nanorods [29]. In other words, the low synthesis temperature should be beneficiary for crystallization of fine nanoparticles while higher temperatures should enable $1 \mathrm{D}$ structures formation. The importance of diffusion process to the ceria nanorods formation has also been stressed by Soykal et al. [30] in discussion on oriented attachment mechanism that allows ceria nanocrystals to grow into a certain direction. Proofs that oriented attachment crystal growth is the main route for the ceria nanorods formation have been presented by several authors $[9,31]$. The fact that temperature is the key parameter for hydrothermal synthesis of $1 \mathrm{D}$ ceria nanostructures has been noted by several authors $[9,29,32]$.

Representative FESEM micrographs of samples CNR5 and CNR6, having the smallest and the greatest SSA, are shown in Figure 4. Sample 6 consists of irregular-shaped and heavily agglomerated nanoparticles. Morphology of sample 5 is similar to that of sample 6 but additionally some $1 \mathrm{D}$ structure could be observed. Sample 5 is prepared at higher temperature $\left(180^{\circ} \mathrm{C}\right)$ so it seems that SEM analysis corroborated assumed relationship between temperature and morphology. In other words, higher temperature enabled the formation of $1 \mathrm{D}$ structures despite being entangled with agglomerated spherical nanoparticles. Fine ceria nanoparticles yield great SSA, while coarser nanorods yield smaller SSA.

The catalytic efficiency of the prepared ceria samples was studied for soot oxidation according to the method of Sudarsanam et al. [25]. Four samples were selected for catalytic test, including samples CNR6 (selected since it has the greatest SSA) and CNR5 (selected since it has the smallest SSA). For comparison, soot was oxidized under the same conditions without ceria. The curves showing percentage of the soot oxidized versus temperature are shown in Figure 5. Regardless of catalyst presence, the oxidation starts around $450^{\circ} \mathrm{C}$. However, from Figure 5 it is obvious that without catalyst the oxidation process is the slowest and ends at the temperature of $995^{\circ} \mathrm{C}$. In samples containing catalyst, soot oxidation rate is greater and process is completed at lower temperatures in a range of 850 to $960^{\circ} \mathrm{C}$. Surprisingly, catalysts with greater SSA (samples CNR6 and CNR3) turned out to be less active than catalysts with smaller SSA (samples CNR5 and CNR8). Importance of morphology and exposed surface crystal plane for catalytic activity of nanocrystalline ceria is often reported $[2,25,33]$. Ceria nanorods seem to show particularly high catalytic activity $[2,34]$. Therefore, it seems that morphology has greater influence on catalytic activity than SSA. However, relative importance of morphology and particle size (and, in turn, SSA) in physical properties and catalytic activity of nanocrystalline ceria is still subject of debate [2].

\section{Conclusions}

Taguchi experimental design has been employed to examine the effect of various hydrothermal synthesis parameters on the specific surface area, as well as on other properties of ceria. Nanocrystalline ceria with SSA as great as $226 \mathrm{~m}^{2} \mathrm{~g}^{-1}$ was prepared which is unprecedented for the hydrothermal synthesis of ceria. It was shown that the only significant factor

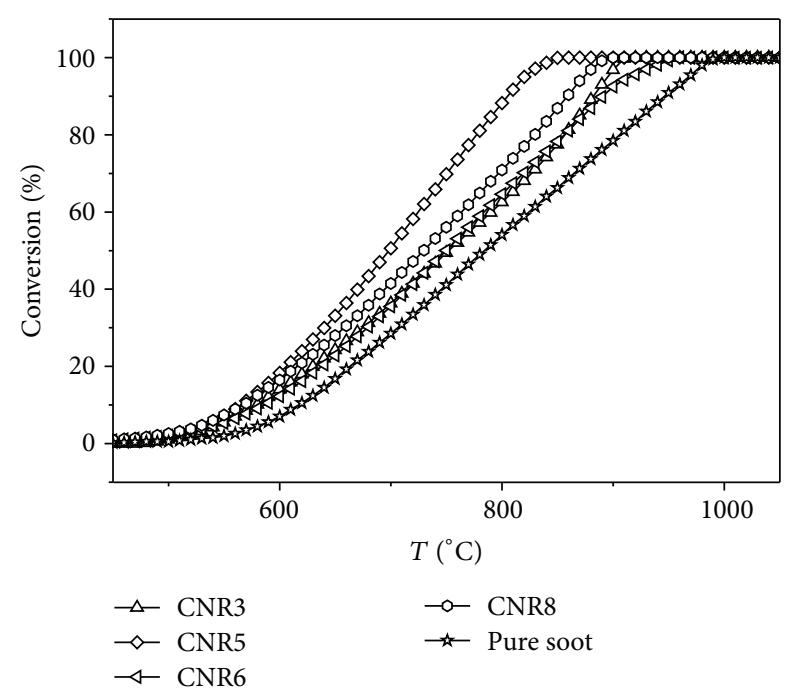

FIGURE 5: Soot conversion versus temperature for samples CNR3, CNR5, CNR6, and CNR8 mixed with soot in ratio of $1: 1$. Pure soot conversion was added for comparison.

for achieving high SSA is the hydrothermal synthesis temperature, where lower temperature yields greater SSA. The reason for such behavior was found in diffusion coefficient which is temperature dependent. When low it favors nucleation, while when high it favors crystal growth and formation of one-dimensional structures. The occurrence of $1 \mathrm{D}$ structure in sample obtained at higher temperature exhibiting the smallest SSA has been confirmed. Increase of SSA in samples was accompanied with proportional decrease of crystallites, to crystallite size as low as $5.9 \mathrm{~nm}$. Samples with smaller SSA turned out to possess better catalytic activity, which is explained with the influence of ceria catalyst nanoparticles morphology, that is, presence of $1 \mathrm{D}$ nanostructural entities.

\section{Competing Interests}

The authors declare that they have no competing interests.

\section{Acknowledgments}

The support of the University of Zagreb Faculty of Chemical Engineering and Technology is gratefully acknowledged. The authors would like to thank G. Matijašić for valuable discussion on adsorption-desorption isotherms.

\section{References}

[1] A. G. Macedo, S. E. M. Fernandes, A. A. Valente, R. A. S. Ferreira, L. D. Carlos, and J. Rocha, "Catalytic performance of ceria nanorods in liquid-phase oxidations of hydrocarbons with tert-butyl hydroperoxide," Molecules, vol. 15, no. 2, pp. 747-765, 2010.

[2] J. M. López, A. L. Gilbank, T. García, B. Solsona, S. Agouram, and L. Torrente-Murciano, "The prevalence of surface oxygen vacancies over the mobility of bulk oxygen in nanostructured 
ceria for the total toluene oxidation," Applied Catalysis B: Environmental, vol. 174-175, pp. 403-412, 2015.

[3] X. Gao, C. Chen, S. Ren, J. Zhang, and D. Su, "Structural effects of cerium oxides on their thermal stability and catalytic performance in propane oxidation dehydrogenation," Chinese Journal of Catalysis, vol. 33, no. 7, pp. 1069-1074, 2012.

[4] H. Jin, N. Wang, L. Xu, and S. Hou, "Synthesis and conductivity of cerium oxide nanoparticles," Materials Letters, vol. 64, no. 11, pp. 1254-1256, 2010.

[5] B. Nematollahi, M. Rezaei, and E. Nemati Lay, "Synthesis of nanocrystalline $\mathrm{CeO}_{2}$ with high surface area by the taguchi method and its application in methanation," Chemical Engineering and Technology, vol. 38, no. 2, pp. 265-273, 2015.

[6] A. Bumajdad, J. Eastoe, and A. Mathew, "Cerium oxide nanoparticles prepared in self-assembled systems," Advances in Colloid and Interface Science, vol. 147-148, pp. 56-66, 2009.

[7] S. Chowdhury, M. Yasir, M. A. B. Bustam, and K.-S. Lin, "Hydrothermal synthesis and characterization of one dimensional ceria nanorod for chromium ion removal from wastewater," Journal of Energy Technologies and Policy, vol. 3, pp. 489494, 2013.

[8] X. Ge, Z. Li, and Q. Yuan, "1D ceria nanomaterials: versatile synthesis and bio-application," Journal of Materials Science and Technology, vol. 31, no. 6, pp. 645-654, 2015.

[9] Z. C. Gernhart, C. M. Marin, J. J. Burke, K. O. Sonnenfeld, and C. L. Cheung, "Additive-free synthesis of cerium oxide nanorods with reaction temperature-tunable aspect ratios," Journal of the American Ceramic Society, vol. 98, no. 1, pp. 39-43, 2015.

[10] N. Ohtake, Y. Yamane, K. Nakagawa, M. Katoh, and S. Sugiyama, "Hydrothermally synthesized ceria with a high specific surface area for catalytic conversion of ethanol to ethylene," Journal of Chemical Engineering of Japan, vol. 49, no. 2, pp. 197203, 2016.

[11] C.-Y. Cao, Z.-M. Cui, C.-Q. Chen, W.-G. Song, and W. Cai, "Ceria hollow nanospheres produced by a template-free microwave-assisted hydrothermal method for heavy metal ion removal and catalysis," Journal of Physical Chemistry C, vol. 114, no. 21, pp. 9865-9870, 2010.

[12] X. Yin, Y. Zhang, Z. Fang, Z. Xu, and W. Zhu, "Hydrothermal synthesis of $\mathrm{CeO}_{2}$ nanorods using a strong base-weak acid salt as the precipitant," Nanoscience Methods, vol. 1, no. 1, pp. 115$122,2012$.

[13] G. Renu, V. V. Divya Rani, S. V. Nair, K. R. V. Subramanian, and V.-K. Lakshmanan, "Development of cerium oxide nanoparticles and its cytotoxicity in prostate cancer cells," Advanced Science Letters, vol. 6, pp. 17-25, 2012.

[14] M. Hirano and E. Kato, "Hydrothermal synthesis of cerium(IV) oxide," Journal of the American Ceramic Society, vol. 79, no. 3, pp. 777-780, 1996.

[15] M. Hirano and E. Kato, "Hydrothermal synthesis of nanocrystalline cerium(IV) oxide powders," Journal of the American Ceramic Society, vol. 82, no. 3, pp. 786-788, 1999.

[16] J. Wang, Q. Liu, and Q. Liu, "Ceria- and Cu-doped ceria nanocrystals synthesized by the hydrothermal methods," Journal of the American Ceramic Society, vol. 91, no. 8, pp. 27062708, 2008.
[17] J. Park, J. Kim, J. Han, S.-W. Nam, and T.-H. Lim, “Hydrothermal synthesis and characterization of nanocrystalline ceria powders," Journal of Industrial and Engineering Chemistry, vol. 11, no. 6, pp. 897-901, 2005.

[18] H.-X. Mai, L.-D. Sun, Y.-W. Zhang et al., "Shape-selective synthesis and oxygen storage behavior of ceria nanopolyhedra, nanorods, and nanocubes," Journal of Physical Chemistry B, vol. 109, no. 51, pp. 24380-24385, 2005.

[19] C. Pan, D. Zhang, and L. Shi, "CTAB assisted hydrothermal synthesis, controlled conversion and $\mathrm{CO}$ oxidation properties of $\mathrm{CeO}_{2}$ nanoplates, nanotubes, and nanorods," Journal of Solid State Chemistry, vol. 181, no. 6, pp. 1298-1306, 2008.

[20] A. I. Y. Tok, S. W. Du, F. Y. C. Boey, and W. K. Chong, "Hydrothermal synthesis and characterization of rare earth doped ceria nanoparticles," Materials Science and Engineering $A$, vol. 466, no. 1-2, pp. 223-229, 2007.

[21] S.-F. Wang, C.-T. Yeh, Y.-R. Wang, and Y.-C. Wu, "Characterization of samarium-doped ceria powders prepared by hydrothermal synthesis for use in solid state oxide fuel cells," Journal of Materials Research and Technology, vol. 2, no. 2, pp. 141-148, 2013.

[22] J. Yang, L. Lukashuk, H. Li, K. Föttinger, G. Rupprechter, and U. Schubert, "High surface area ceria for CO oxidation prepared from cerium t-butoxide by combined sol-gel and solvothermal processing," Catalysis Letters, vol. 144, no. 3, pp. 403-412, 2014.

[23] Y. Kamimura, M. Shimomura, and A. Endo, "Simple templatefree synthesis of high surface area mesoporous ceria and its new use as a potential adsorbent for carbon dioxide capture," Journal of Colloid and Interface Science, vol. 436, pp. 52-62, 2014.

[24] H. Mohamed, M. Hisyam Lee, M. Sarahintu, S. Salleh, and B. Sanugi, "The use of Taguchi method to determine factors affecting the performance of destination sequence distance vector routing protocol in mobile ad hoc networks," Journal of Mathematics and Statistics, vol. 4, no. 4, pp. 194-198, 2008.

[25] P. Sudarsanam, B. Hillary, D. K. Deepa et al., "Highly efficient cerium dioxide nanocube-based catalysts for low temperature diesel soot oxidation: The cooperative effect of cerium- and cobalt-oxides," Catalysis Science and Technology, vol. 5, no. 7, pp. 3496-3500, 2015.

[26] M. Thommes, K. Kaneko, A. V. Neimark et al., "Physisorption of gases, with special reference to the evaluation of surface area and pore size distribution (IUPAC Technical Report)," Pure and Applied Chemistry, vol. 87, no. 9-10, pp. 1051-1069, 2015.

[27] I. V. Zagaynov and S. V. Kutsev, "Formation of mesoporous nanocrystalline ceria from cerium nitrate, acetate or acetylacetonate," Applied Nanoscience, vol. 4, no. 3, pp. 339-345, 2014.

[28] P. J. Ross, Taguchi Technique for Quality Engineering, McGrawHill, New York, NY, USA, 1988.

[29] C. Sun, H. Li, H. Zhang, Z. Wang, and L. Chen, "Controlled synthesis of $\mathrm{CeO}_{2}$ nanorods by a solvothermal method," Nanotechnology, vol. 16, no. 9, pp. 1454-1463, 2005.

[30] I. I. Soykal, H. Sohn, B. Bayram et al., "Effect of microgravity on synthesis of nano ceria," Catalysts, vol. 5, no. 3, pp. 1306-1320, 2015.

[31] Z. Yang, K. Zhou, X. Liu, Q. Tian, D. Lu, and S. Yang, "Singlecrystalline ceria nanocubes: size-controlled synthesis, characterization and redox property," Nanotechnology, vol. 18, Article ID 185606, 2007. 
[32] P. X. Huang, F. Wu, B. L. Zhu et al., " $\mathrm{CeO}_{2}$ nanorods and gold nanocrystals supported on $\mathrm{CeO}_{2}$ nanorods as catalyst," Journal of Physical Chemistry B, vol. 109, no. 41, pp. 19169-19174, 2005.

[33] S. Chowdhury and K.-S. Lin, "Synthesis and characterization of ID ceria nanomaterials for CO oxidation and steam reforming of methanol," Journal of Nanomaterials, vol. 2011, Article ID 157690, 16 pages, 2011.

[34] K. Zhou, X. Wang, X. Sun, Q. Peng, and Y. Li, "Enhanced catalytic activity of ceria nanorods from well-defined reactive crystal planes," Journal of Catalysis, vol. 229, no. 1, pp. 206-212, 2005. 

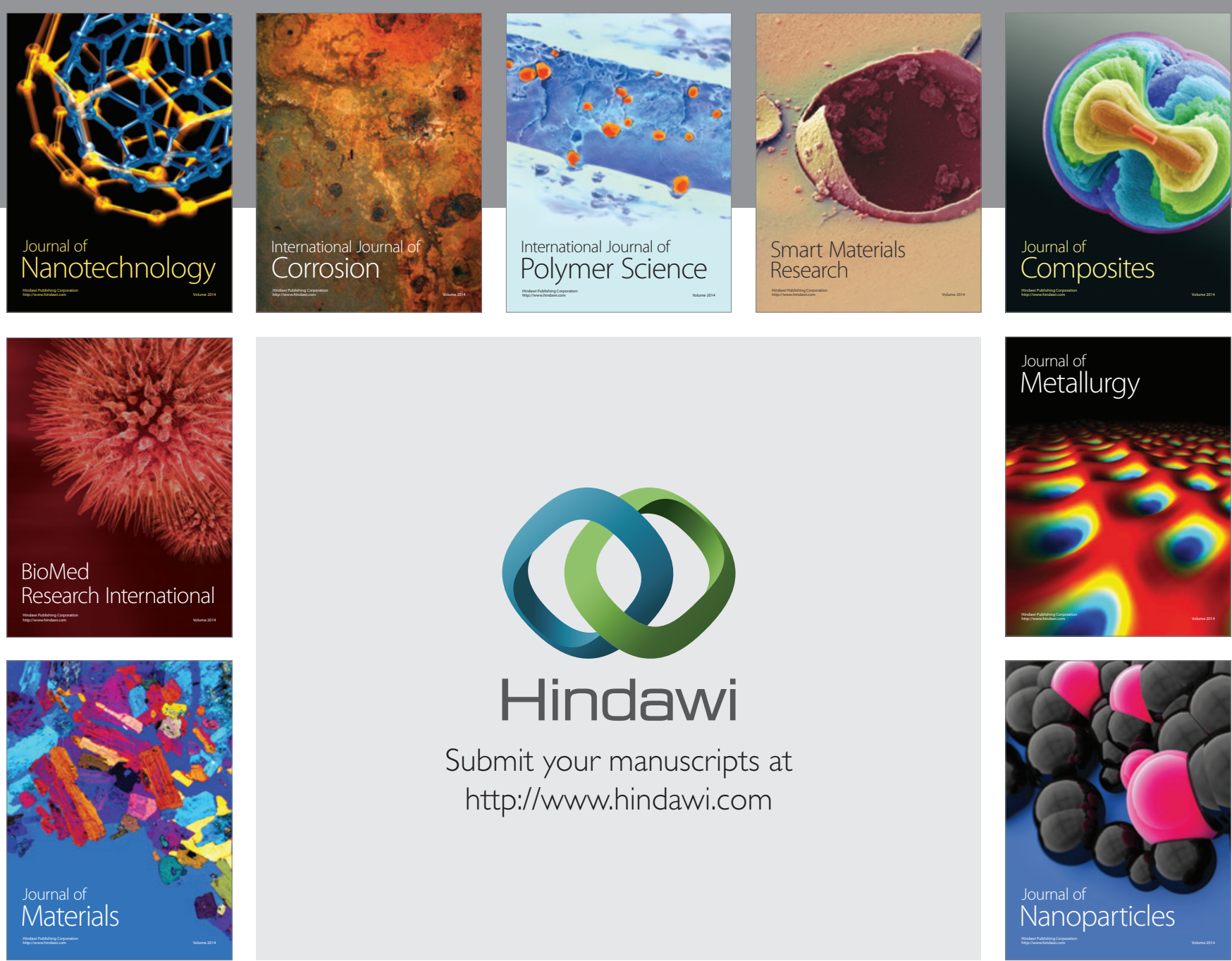

\section{Hindawi}

Submit your manuscripts at

http://www.hindawi.com

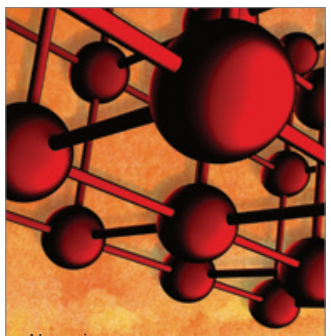

Materials Science and Engineering
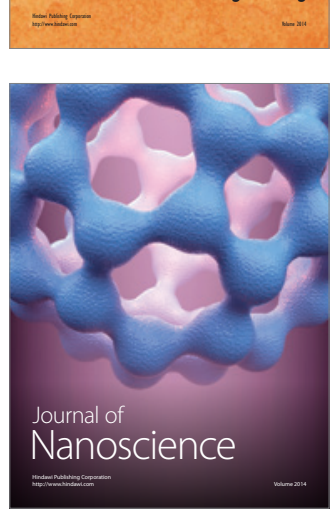
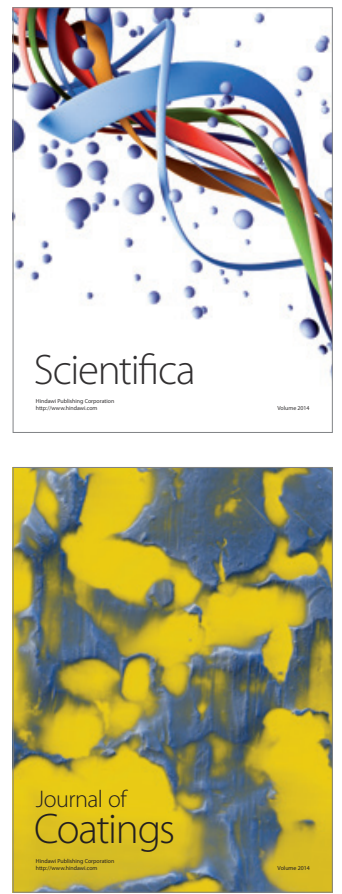
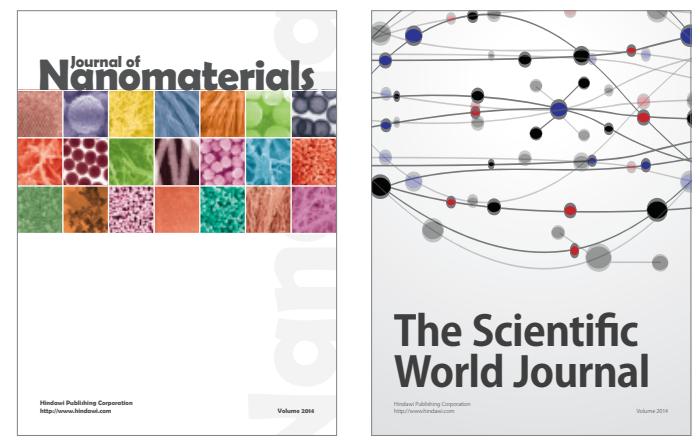

The Scientific World Journal
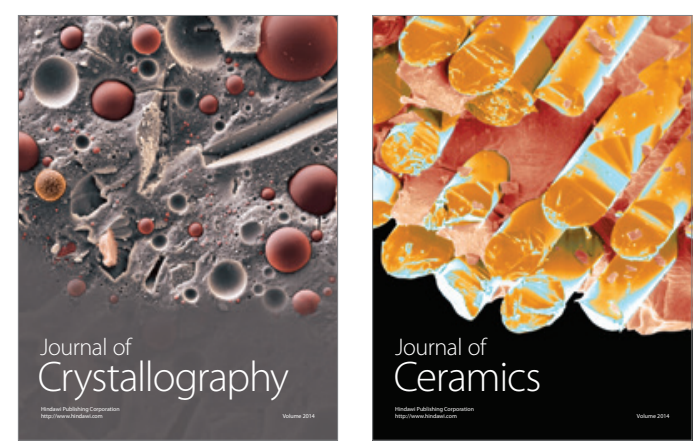
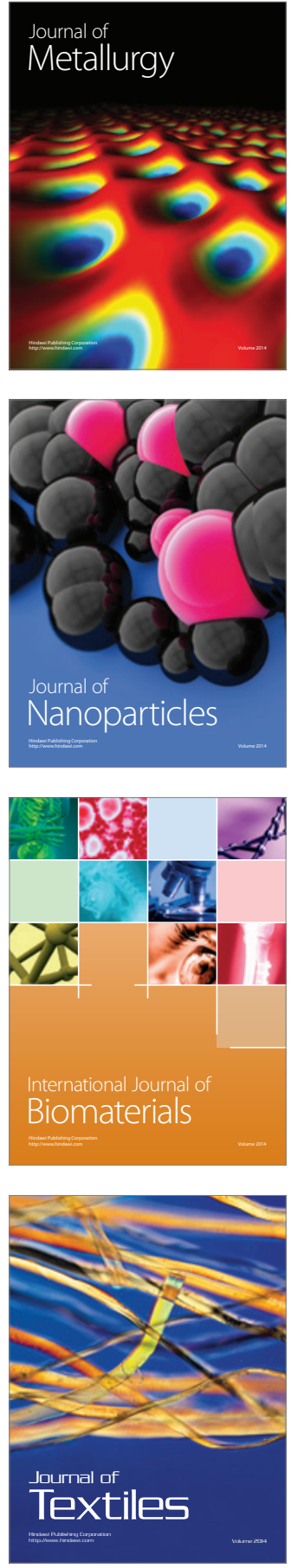